\title{
Cecilia Lazzeretti*
}

\section{A Landscape Never Goes Out of Style Diachronic Lexical Variation in Exhibition Press Announcements}

\begin{abstract}
The paper focuses on diachronic lexical variation in a professional textual genre which has gained growing importance over time in the field of museum public relations and art discourse: exhibition press announcements (EPAs). The aim of the analysis is to investigate the language of EPAs from a diachronic perspective in order to identify word frequencies showing large increases or decreases, or stability in word frequencies. Baker's (2011) method to distinguish variation over time across multiple corpora was applied and particular attention was placed on the presence of "lockwords", i.e. words "relatively static in terms of frequency" (Baker 2011: 66). The analysis is carried out on a corpus of EPAs dating from 1950 to 2009 issued by American and British museums. The study reports on a number of trends relating to linguistic and cultural change of EPAs, including the emergence of new criteria in assessing the value of artists and artworks despite a certain consistency in terms of subjects, the shift from one-item to multi-item exhibitions and the preference for more vivid and straight-forward descriptions. For instance, the frequency of the noun landscape has remained stable over time, suggesting that this subject is particularly consistent in art displays, quite a sort of classic, that never grows old, while the artist's career - a word showing a clear pattern of growth - has become particularly valuable over time for museum professionals in charge of exhibitions.
\end{abstract}

Keywords: press releases, art discourse, museums, diachronic, variation, lexis

\section{Introduction}

Despite their undoubted affiliation to the textual genre of press releases, it would be limiting to present exhibition press announcements (EPAs) as a mere subgenre of this category. The high level of creativity characterizing EPAs, both in terms of lexical choices and structure, their strong promotional intent, often realized through a massive use of evaluative language and emotional linguistic features, their capacity to address media people as well as the lay public through their e-dimension (Lazzeretti/Bondi 2012), encourage readers to consider them as a genre worth of interest per se, sharing their own peculiar features, as well as their own rules.

As part of the press materials daily issued by a museum's press office, EPAs are meant for a restricted category of journalists, those in charge of arts reporting and criticism. In the last decades, they have become crucial for cultural institutions in terms of communication with their audiences, especially in consequence of the development of thematic exhibitions characterized by strong educational goals and aiming to appeal to a wide range of visitors (McManus 2000, Schiele 1995, Jacobi/Poli 1995), a phenomenon that can be dated back to the 1940s and which is still on-going. EPAs reflect therefore the value system of the professional communities involved in this environment: on the one hand, experts in charge of the organization and promotion of art exhibitions, such as curators, managers and press officers, and, on the other hand, media people interested in art news reports and reviews.

Among the reasons for the peculiarity of EPAs is their positioning in the middle ground of two different discourse domains: firstly, news (or media) discourse, which is mainly informative,

\footnotetext{
* Cecilia Lazzeretti

Scuola di Dottorato in Scienze Umanistiche

c/o Dipartimento di Studi Linguistici e Culturali

Università di Modena e Reggio Emilia

Via dell'Università, 4, Modena

cecilia.lazzeretti@unimore.it
} 
and secondly, for their content specifically related to art, art discourse, whose main communicative purpose is descriptive and evaluative. The latter, in particular, plays a very important role in shaping EPAs as they are, because it allows us to differentiate them from common press releases.

The connection between EPAs and art discourse still needs to be fully explicated. In the first place, there is a connection at the level of contents: in announcing up-coming exhibitions, EPAs indulge in detailed descriptions of the artworks to be exhibited, in the techniques involved, and in the exhibiting space design. The featured artists are presented through their biographies and the mention of their most important works, as well as the artistic movements relevant for the exhibition. Description, to be considered as "an attention-managing device that the writer uses to direct the reader's attention to new referents" (Bondi 2013: 7), has to be regarded as a key concept for EPAs.

Moreover, the professional environment to which EPAs belong is deeply permeated by art discourse(s): EPAs are "texts in museums" (Ravelli 2006:2) which function as communication tools between the institutions and their audiences. They are especially written for art journalists in charge of reviews and criticism, although, once they have been published on museums websites, they are also able to reach a general audience (Lazzeretti and Bondi 2012). Moreover, they can be considered prime examples of what scholars have been alternatively calling 'artwriting' (Carrier 1987), 'artspeak' (Atkins 1990, Harris 2003), 'art talk' and 'artworld discourse' (Irvine 2004-2009).

As is so often the case for concepts that stand for complex phenomena, the notion of art discourse is essentially fuzzy and the plurality of terminology mirrors the difficulty in defining it. Harris (2003) adopted the term 'artspeak', previously popularized by the art historian Atkins (Atkins 1990), "not simply to include the buzzwords used by critics in certain sectors of the art world, but to cover the whole range of discourse about works of art and their appreciation (or disparagement)" (Harris 2003: xii). The meaning of the term goes beyond what Carrier called 'artwriting', which was restricted to "texts by both art critics and art historians" (Carrier 1987: 141).

Irvine (2004-2009) prefers the term 'artworld discourse', which he defines as a "distributed network system of ways of talking" about art, comprising " the various vocabularies, arguments, professional fields, and institutionalized contexts for making statements". The aim of the multiple discourses employed within the artworld is to describe, talk and argue about art objects and to identify 'art' in itself. As Irvine (2004-2009) puts it, artworld discourse is "a function of the artworld's role in defining the cultural category of art and maintaining the art/non-art binary". Irvine's network of artworld discourse comprises: a) mainstream press, b) blogs and quoted popular discourse, c) independent weeklies and websites, d) weekly magazines that include art 'coverage', e) art magazines and monthly art press (and their websites), f) advertising in the magazines, press, and websites, g) curatorial discourse in museum publications, catalogues, and exhibition texts, h) gallery publications, catalogs, press releases, $\mathrm{j}$ ) academic and scholarly books and articles.

Studying art discourse in one of its professional applications and the specific case of EPAs can tell us much about how art is communicated through language. Moreover, adopting a diachronic perspective allows us to gain a significant insight into lexical variation over time and, more broadly speaking, into the linguistic evolution of art discourse.

The purpose of the present research is to examine how the language of EPAs has developed over the past six decades and how art has been presented to the press in the same time lapse. To this purpose, a corpus of EPAs dating back to 1950 onwards was explored in order to identify any increase, decrease or stability in word frequencies. The enquiry addresses the following research questions:

- What lexical variation can be identified in EPAs from the 1950s up to now?

- Has there been any language change reflecting socio-cultural change, for instance in the way exhibitions are organized and subjects are selected? 
After reviewing existing literature, the present article outlines the design of the corpus (section 3), as well as the methods applied in the analysis (section 4). In section 5, the results of the analysis are presented, while conclusions are drawn in section 6.

\section{Literature review}

According to Baker (2011), "corpus approaches to diachronic change are still in their infancy (and have often only compared two time periods), and it is only recently, with the development of multiple sets of comparable reference corpora, that we can start to trace lexical change over time" (2011:66). Similarly, Partington (2012) defines modern diachronic corpus-assisted discourse studies (MD-CADS) as a nascent discipline, characterised by the novelty of methodology and topics: "It employs large corpora of a parallel structure and content from different moments of contemporary time in order to track changes in modern language usage but also social, cultural and political changes over modern times, as reflected in language" (2012: 51).

It comes therefore as no surprise that studies on media language from a diachronic perspective are relatively rare. For instance, a diachronic analysis of press releases - a genre born at the beginning of the last century ${ }^{1}$ - has not been carried out yet. The same has to be noted with regard to modern diachronic studies especially devoted to art discourse.

Among scholars involved in modern diachrony applied to media discourse are Hundt and Mair (1999), who, in their tracking of changes in newspaper prose between 1960 and 1990, noted a greater use of contractions, and first- and second-person pronouns, where these oral features were adopted in an attempt to appeal to a wider reading audience. Partington $(2010,2012)$ and Duguid (2010) have also been working on British broadsheet newspapers, as collected in the SiBol (1993) and SiBol (2005) corpora. Duguid (2010) has pointed out that the language of newspapers has changed over time in terms of an increasingly more conversational and informal style, along with a notable increase in a particular kind of evaluative and promotional language, as a result of a proportional increase in soft news, supplements and reviews.

Baker's studies $(2010,2011)$ carried out across multiple corpora are particularly relevant in methodological terms for the present work. Baker (2011) investigated four equal-sized reference corpora of written British English from 1931, 1961, 1991, and 2006, in search of patterns of vocabulary change and stability. He also considered several methods to identify variation over time and categorized words as showing sharp frequency increases, decreases, or as remaining stable. He called the latter "lockwords", because they are "relatively static in terms of frequency" (Baker 2011: 66) and "appear to be 'locked' in place" (ibid. 73). Finally, he reported on a number of trends relating to language (specifically British English) and culture change, including a tendency for written language to become less verbose, more informal and personal. In a previous study, Baker (2010) carried out a corpus-based comparison of gendered terms (male and female pronouns, gender-related nouns, and terms of address) across the same four corpora, finding out that while there had been reductions in some gender stereotypes, others were maintained: males, in particular, were referred to more often than females. Both studies show the value of using corpus methods in order to investigate change in the frequency and context of use of specific items of language over time.

\section{Materials}

As none of the existing diachronic corpora was suitable for the purposes of this research, a new corpus was compiled for this study: the EPA Diacorpus. This is a diachronic corpus, made up of a total of 299,138 words (tokens), consisting of various subcorpora. It includes 378 EPAs, half is-

1 In 1906, after the Atlantic City train wreck, Ivy Lee issued what is often considered to be the very first press release in history, convincing the company Pennsylvania Railroad to openly disclose information to journalists, before they could hear information from elsewhere. 
sued by American museums and half by British museums, dating from 1950 to 2009, as summarized by the following table (1):

\begin{tabular}{|l|r|}
\hline \multicolumn{2}{|c|}{ EPA Diacorpus } \\
\hline Corpus size & 299,138 words \\
\hline Number of texts & 378 \\
\hline Full texts & Full texts \\
\hline Medium & Written (press releases) \\
\hline Subject & $\begin{array}{r}\text { Exhibition press } \\
\text { announcements }\end{array}$ \\
\hline Authorship & $\begin{array}{r}\text { Multiple professional } \\
\text { writers }\end{array}$ \\
\hline Language & $\begin{array}{r}\text { Texts written in English } \\
\text { by native speakers }\end{array}$ \\
\hline Publication date & $\begin{array}{r}\text { March 1950- } \\
\text { December 2009 }\end{array}$ \\
\hline
\end{tabular}

Table 1. Features of EPA Diacorpus

A corpus of this size may be small compared to the multi-million-word corpora available today, but for the purposes of the present study, it was assumed to be balanced and representative.

EPAs were randomly and evenly selected across decades, with no particular criteria beside their status of exhibition press announcements, e.g. press releases announcing an art show. All other press releases issued by museums, such as ordinary news, announcements of artist talks, presentations of films or books, accomplishments, awards, new appointments, philanthropic events, etc, were dismissed. It is to be noted, however, that EPAs represent the largest part of the documents usually produced by museum press offices.

The number of writers involved in the composition of EPAs is not easy to quantify. Although most of the EPAs collected in the corpus are signed by a press officer - an acknowledged professional specialized in museum public relations - EPAs are often the result of the work of a composite team of experts. A first draft, for instance, may be traced back to a text written by the curator of an exhibition, who first conceived a project. The EPA also has to be verified by members of the managerial staff - i.e. the director of the museum, the board of directors, etc...-, while other useful comments and suggestions may come from co-workers, before the final draft is released. Therefore, the EPA Diacorpus represents multiple writers.

Furthermore, the EPA Diacorpus is a DIY - 'do-it-yourself' - corpus (McEnery et al. 2006: 71), which is built on the following methodological principles, with respect to both the choice of museums and the period selection for inclusion. The decision whether to create a hybrid corpus, including both British and American EPAs, or a more homogeneous corpus, comprised of only American (or only British) EPAs, was also crucial.

With regard to the first aspect, large, high-profile museums were preferred, because they could guarantee a significant coverage of EPAs across the twentieth century and their accessibility for research. The selection was also influenced by the format of the data, which was available in electronic form only in a minor part. Museums began to digitalize press releases at the end of the 1990s or even later. The New York Museum of Modern Art ${ }^{2}$ and the Los Angeles J. Paul Getty Museum $^{3}$ provide a significant exception, allowing website visitors to search within their digitalized historical archives updated respectively from 1929 and from 1954 onwards. The New York Solomon R. Guggenheim Museum ${ }^{4}$ also offers an overview of historical press releases dating

2 http://www.moma.org/learn/resources/press archives

3 http://www.getty.edu/research

4 http://www.guggenheim.org/new-york/collections/library-and-archives/archive-collections/A0035/ 
from 1952 onwards on its website, but the work is still in progress and at the moment only a small number of documents has been digitalized. Furthermore, British museums retain historical EPAs only on paper format and documents have to be consulted in loco. As a result, most British documents of the EPA Diacorpus, especially the earlier, were found directly at the museums archives; they were photographed, OCR scanned and transformed into *.txt format. A further amount of EPAs, already available in digital form, was downloaded from websites. In particular, earlier British EPAs, dating from 1950 to 1999, were found at the London National Gallery Library, the London Royal Academy of Arts Library and the London Victoria and Albert Museum Archives. Some US institutions also cooperated in a significant way sending paper or digital copies of historical EPAs from their archives (The New York Frick Collection and The Chicago Museum of Contemporary Art).

The sources of the EPA Diacorpus, the number of EPAs retrieved for each institution and the original format of documents are summarized in the table below (Tab. 2):

\begin{tabular}{|l|l|l|l|l|}
\hline Country & City & Institution & EPAs & Original format \\
\hline UK & London & The Victoria and Albert Museum & 61 & $15 \%$ digital; 85\% on paper \\
\hline UK & London & Royal Academy of Arts & 58 & $35 \%$ digital; 65\% on paper \\
\hline UK & London & National Gallery & 72 & $15 \%$ digital; 85\% on paper \\
\hline US & Los Angeles & J. Paul Getty Museum & 35 & $100 \%$ digital \\
\hline US & New York & Museum of Modern Art & 74 & $100 \%$ digital \\
\hline US & New York & The Solomon R. Guggenheim Foundation & 46 & $100 \%$ digital \\
\hline US & Chicago & Museum of Contemporary Art & 17 & $100 \%$ digital \\
\hline US & New York & The Frick Collection & 15 & $100 \%$ on paper \\
\hline & & TOT. & 378 & \\
\hline
\end{tabular}

Table 2. EPA Diacorpus: sources, number and original format of EPAs

With regard to the second aspect, i.e. the period to cover for the present diachronic study, six decades were selected, from the start of regular publication of EPAs in 1950 to 2009. Earlier examples may also be identified ${ }^{5}$, but their distribution was not homogeneous across decades, especially during World War II, when most museums interrupted their activities. Moreover, some highly representative museums, such as the New York Solomon R. Guggenheim Museum and the Los Angeles J. Paul Getty Museum, were only founded in the 1950s.

As for the third aspect - whether to create a corpus made up only of British EPAs, or only of American EPAs, or a hybrid one - the last option was decided upon. This decision was taken in view of the fact that American and British EPAs share much similarity and can be studied together. According to MacLean (1997) the development of museum public relations - and therefore EPAs - started in the US after World War II and subsequently spread in Western Europe. It can therefore be assumed that American EPAs inspired the British ones. British and American EPAs have been evenly distributed in the EPA Diacorpus and together they can give a representative linguistic picture of the genre and of the period they cover.

Nevertheless, the EPA Diacorpus is divided into two main components, US EPAs and UK EPAs, to be analysed separately and together. Each component is further divided into six sub-cor-

5 The first press release issued by a museum which was found available dates back to 1929 and belongs to the New York Museum of Modern Art. It announces the opening of the museum and the start of its activities: "The belief that New York needs a Museum of Modern Art scarcely requires apology. All over the world the rising tide of interest in the modern movement has found expression not only in private collections but also in the formation of great public galleries for the specific purpose of exhibiting permanent as well as temporary collections of modern art. That New York has no such gallery is an extraordinary anachronism. " (Publicity for organization of Museum, August 1929, MoMA press archives. Accessed at http://www.moma.org/learn/resources/press_archives). 
pora, one for each decade, which evenly comprises both British and American EPAs, as shown in the table below (Tab. 3).

\begin{tabular}{|l|l|l|l|}
\hline & US & UK & TOT \\
\hline 1950 & 30 & 30 & 60 \\
\hline 1960 & 30 & 32 & 62 \\
\hline 1970 & 33 & 33 & 66 \\
\hline 1980 & 30 & 31 & 61 \\
\hline 1990 & 31 & 32 & 63 \\
\hline 2000 & 33 & 33 & 66 \\
\hline & 187 & 191 & 378 \\
\hline
\end{tabular}

Table 3. EPA Diacorpus: number of press releases across decades and countries

\section{Methods}

The present study starts from a "naive" position, allowing data to drive the research and adopting what Tognini-Bonelli (2001) refers to as a 'corpus-driven' approach. Baker's (2011) method to observe diachronic change across multiple corpora and to distinguish variation over time served as a framework.

Following Baker (2011), it was stipulated that for a word to be of interest in terms of diachronic variation, it would need to occur at least 100 times when its frequencies across all the six decades $(1950,1960,1970,1980,1990,2000)$ were added together. Three hundred and twenty-six words met this criterion. In view of the size of the corpus, totaling 300 thousand words, the relevant proportion is about $1 / 3,000$.

Using the "detailed consistency analysis" function in Wordsmith Tool 5 (Scott 2007), it was possible to obtain a single table that showed every instance of each word in all six sub-corpora, along with their frequencies.

After dismissing a number of possible measures to quantify the strength of difference between word frequencies, such as the chi-square test and the Pearson correlation coefficient, Baker (2011) took into consideration Hofland and Johansson's (1982) method. Hofland and Johansson compared word frequencies in the British LOB and American Brown corpora using the following formula:

(1) $\frac{\text { Freq LOB }- \text { Freq Brown }}{\text { Freq LOB }+ \text { Freq Brown }}$

Baker (2011) tried adapting this measure to see if it would be effective on his four corpora:

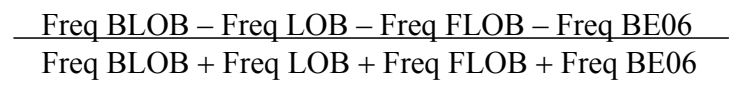

I followed this suggestion and adapted Hofland and Johannson's measure to the six sub-corpora of the EPA Diacorpus in this way:

$$
\frac{\text { Freq } 1950-\text { Freq } 1960-\text { Freq } 1970-\text { Freq } 1980-\text { Freq } 1990-\text { Freq } 2000}{\text { Freq } 1950+\text { Freq } 1960+\text { Freq } 1970+\text { Freq } 1980+\text { Freq } 1990+\text { Freq } 2000}
$$

This gave a score for each word between 0.07 and -1 . The twenty words with the highest score, showing therefore the largest decrease over time, were $m r s, m r$, picture, represented, man, acquired, shown, here, had, shows, exhibited, william, painters, examples, three, so, paris, wednesday, still and architecture. Conversely, the twenty words with the lowest scores, showing the largest increases over time, were office, career, tel, $u k$, org, www, admission, tickets, frick, getty, images, photography, visual, catalogue, please, students, contact, events, tour and $v \& a$. However, 
the adapted formula of Hofland and Johannson does not show real trends because it does not deal with exceptional data appropriately, as in the case of irregular frequency profiles across the six decades, where a clear increasing or decreasing pattern cannot be detected. This measure therefore had to be refined with a further statistical method. Among several bottom-up approaches discussed by Hilpert/Gries (2009), Kendall's tau coefficient appears as a wide-spread measure for correlations, which, correlating the sequence of corpus sub-periods with the frequencies of each word, produces correlation coefficients for each example. A value close to 0 indicates the absence of a trend, while values approaching either 1 or -1 indicate that the passage of time correlates perfectly with an increase or decrease in frequency respectively. Kendall's tau coefficients were calculated with the aid of a statistical software integrated into the Microsoft Excel application, Analyse-it, which allows users to automatically determine several types of correlation, Kendall included. The requirements of the test are two variables measured on an ordinal or continuous scale. Data in Excel worksheets were therefore arranged accordingly, inserting two variables for each word in the dataset: in the first column the frequencies of the item across the six decades and in the second column the sequence of the EPA Diacorpus sub-periods. In the case of the noun picture, for instance, the variables introduced were 96, 84, 16, 1, 15, 12 and 1, 2, 3, 4, 5, 6 respectively. The Kendall's tau value obtained after running the test was -0.7 .

According to Kendall's tau coefficient, the top twenty words showing a downward trend, with a value comprised between -0.9 and -0.5 , are country, $m r$, picture, van, man, here, acquired, famous, chicago, shows, william, shown, had, west, since, i, mrs, represented, exhibited and so. Conversely, the top twenty words showing the highest upward trend, with a value comprised between 0.6 and 1, are tel, office, photography, students, tickets, catalogue, v\&a, uk, career, admission, images, please, contact, tour, org, www, getty, events, frick and visual.

As pointed out by Baker (2011), Hofland and Johansson's method does not reveal which words remained stable over time. Baker suggests using the standard deviation (SD) score to identify high and low variation over time of the frequencies of each word: "Potentially [...], words with a high standard deviation would have changed a great deal in frequency over time, whereas those with low standard deviations would be more stable (2011:72)" by calculating the standard deviations of the 326 words occurring in the corpus at least 100 times when their frequencies across all the six decades $(1950,1960,1970,1980,1990,2000)$ were added together using Excel (function STDEV). The SDs of these words ranged from 3.08 to 1,287.50. The twenty words with the largest standard deviations were exhibition, art, getty, works, museum, guggenheim, new, arts, collection, royal, please, org, $w w w, m r$, academy, press, information, curator, admission, artists, while those with the lowest were landscape, colour, selected, death, held, release, form, used, selection, seen, department, working, chicago, recent, born, abstract, subject, painter, europe and forms. Although interesting, this data needed to be corrected, since SD measures frequency rather than variation. As suggested by Baker (2011), the correction used was the coefficient of variance $(\mathrm{CV})$, calculated by dividing the standard deviation by the mean and then multiplying by 100 . As the CV score does not correlate with frequency, it was used to determine which words had strong and weak variation over time. Next, the word list was divided into three equal-sized portions, reflecting high change, medium change, and low change. The top third of the word list, ordered by $\mathrm{CV}$, was considered to have relatively high variation, the bottom third to have relatively low variation, and the middle third to be relatively indistinctive and was not examined any further. The words ranged in $\mathrm{CV}$ from a minimum of 10.23 , marking the lowest variation, to a maximum of 232.49, marking the highest.

The ten words with highest relative variation were $w w w$, org, $u k$, events, tickets, frick, getty, please, $v \& a$, and tour. Those with the lowest were release, landscape, until, national, which, be, department, seen, form, and drawings. The strongest noun lockwords in the EPA Diacorpus are release, having a frequency profile of $41,53,54,50,51,45$, and landscape, with a frequency profile of $25,27,21,23,28,29$. 
Having identified words that showed real trends of decline, growth, or stability, a selection of words was ranked within the top twenty of each category and, with the aid of Wordsmith Tools 5 (Scott 2007), multiple concordance searches and collocational analyses of these words were conducted to elicit contextual information that might explain their patterns of usage.

In order to compare data, a reference corpus was needed: the comparison corpus in this study was the Time Magazine Corpus (Davies 2007), made up of roughly 100 million words and 275,000 articles taken from the American periodical TIME Magazine (1923 to the present). Freely available on line, it is the largest diachronic corpus of 20th century American English, and its size allows for accurate analysis of linguistic change across the decades.

\section{Growth, decline and stability: the most distinctive findings}

The twenty words that show the strongest frequency increase across the six time periods $(1960$, 1970, 1980, 1990 and 2000) are: tel, office, photography, students, tickets, catalogue, $v \& a, u k$, career, admission, images, please, contact, tour, org, www, getty, events, frick and visual. On the other hand, the twenty words that show the sharpest decreases over time are country, mr, picture, van, man, here, acquired, famous, chicago, shows, william, shown, had, west, since, $i$, mrs, represented, exhibited and so.

Finally, a further list of words showing very little change over time was compiled. The twenty words with the smallest CV score (i.e. with less variation in their frequencies across decades) were: release, landscape, until, national, which, be, department, seen, form, drawings, held, great, known, paintings, among, made, may, first, selected and some.

Due to limited space, not all words in the list will be discussed. Instead, since this paper is narrowly focused on a specific question (the link between language variation of EPAs over time and macro-cultural change in museum settings), the following analysis takes into account only a few words, which have been selected in view of their potential of mirroring cultural change related to museums and art in general: career, visual and photography (increased); picture, shown and shows (decreased); landscape, drawings and paintings (stable).

\subsection{Increasing words: career, visual, photography}

\begin{tabular}{|r|r|r|r|r|r|r|r|}
\hline Word & \multicolumn{1}{|c|}{1950} & \multicolumn{1}{c|}{1960} & \multicolumn{1}{c|}{1970} & \multicolumn{1}{c|}{$\mathbf{1 9 8 0}$} & \multicolumn{1}{c|}{$\mathbf{1 9 9 0}$} & \multicolumn{1}{c|}{$\mathbf{2 0 0 0}$} & \multicolumn{1}{c|}{ Total } \\
\hline career & 0 & 11 & 8 & 27 & 34 & 49 & 129 \\
\hline visual & 2 & 16 & 15 & 14 & 22 & 38 & 107 \\
\hline photography & 4 & 22 & 32 & 59 & 71 & 80 & 268 \\
\hline
\end{tabular}

Table 4. Profile across decades of increasing words: career, visual, photography

\subsection{1. career}

Showing a clear pattern of growth across decades $(0,11,8,27,34,49)$, the word career is one of the most interesting cases of the EPA Diacorpus. It has 129 overall occurrences, quite evenly distributed between the American (71) and British (58) sections. The Time Magazine Corpus was explored for comparison, in order to find out if the word gained increasing importance over time in the language of general media as well, but the result was reversed: career shifted from 1,904 occurrences in the year 1950 to 1,022 occurrences in the year 2000. The increasing trend of career seems therefore a distinct feature of the EPA Diacorpus.

A manual inspection of concordances shows that the career in question is always that of the artists featured in the exhibition. The most frequent three-word clusters associated with career in the corpus are of his career (where the adjective his refers anaphorically or cataphorically to the artist), of the artist's career, the artist's career and throughout his career. The latter cluster intro- 
duces the idea of a long-lasting progress through the artist's lifework, which is also stressed by other evaluative expressions, as in the following concordances (1):

(1) covering all stages of Mirò's long career will be included, extraordinarily rich and prolific career. On view through May 2, every phase of his seventy-year career. Wright is shown to be

In some rarer cases, the artist's career may be qualified as early or brief, due to biographical circumstances, but the semantic prosody of the term sounds positive:

(2) Schiele, and, in an even briefer career, created such dramatic work from the artist's early career stand out as of particular

Although the positive semantic prosody associated with the noun career may seem obvious, the reason for the increasing use of the term over time is worth investigating. We would perhaps expect the frequency of other positively evaluated and career-related words to increase as well in the EPA Diacorpus. Other words from the frequency list that convey positive meaning, especially with regard to the life-work of an artist, are the nouns master and success, and the adjectives $f a-$ mous, known and great. Master and success do not even fall within the cut-off of the 326 most frequent words in the corpus, since they have less than 100 occurrences across all decades. They are therefore indistinctive in terms of variation, due to their limited presence. Conversely, famous has a more relevant presence in the corpus, with a total of 142 occurrences, but a gradually decreasing profile across decades $(35,32,16,19,26,13)$, whereas the adjectives great and known are not only stable, but also quantitatively relevant. Great ( 235 occurrences) is the $12^{\text {th }}$ word showing less variation across decades according to its $\mathrm{CV}$ score and can therefore be classified as a lockword. Similarly, known (202) ranks $13^{\text {th }}$ among lockwords, with a profile across decades of 41 , $39,26,34,24,38$.

A close examination of the concordances related to these more interesting words (famous, great and known) was required. Only 16 occurrences of the adjective famous out of 142 were clearly related to artists, since the majority describe their work, a collection, or, in a smaller number of cases, it defines a group of people (the famous) often portrayed by art. Similarly, only 27 occurrences of the adjective great out of 235 directly describe artists, while most of the instances address art works, art movements, collections and exhibitions. The adjective known is no exception to this pattern, with only a third of the total occurrences (202) related to artists, especially those combined with adverbials, such as well known, best known, internationally known, little known and lesser known.

A first conclusion could be that any evaluation in terms of fame and greatness is preferably addressed to art works and to the relevant artistic context in general, rather than to the artist himself as a person. This tendency is quite stable across decades, although the use of the adjective famous is decreasing, maybe because of its controversial semantic prosody. After Andy Warhol's 1968 quote ("In the future, everyone will be world-famous for 15 minutes") the idea of fame, especially with regard to art, began to carry some disregarded aspects, such as the dissipation of hierarchies and its logical extension that everybody could be famous, and not merely those individuals really worthy of fame (Buchloh 2001).

Conversely, the idea of career as an asset strictly related to the artist became more and more valuable over time, to the point that it can give a justifying reason for the exhibition itself. In other words, we might say that the artist's career has become a preliminary condition for giving him space within a museum and celebrating his work with an exhibition. Youth, as a consequence, does not seem to be particularly valuable in this regard, and for a young artist it is very difficult to emerge in the art scene (see McCarthy et al. 2001). The EPA Corpus confirms the tendency, with less than 10 instances of the expression young artist/s, 18 occurrences of the noun youth and the hapax legomenon youthful. 


\subsection{2. visual}

Totaling 107 occurrences within the EPA Diacorpus, the adjective visual shows a consistently increasing profile across the decades: $2,16,15,14,22,38$. If we take a look at the concordances, we find that the most frequent three-word cluster is the visual arts with 19 occurrences. The first appearance of the cluster dates back to 1961 and belongs to an American EPA. Starting from 1969, the cluster is frequently used in British EPAs as well. See for instance the following examples (3 and 4):

(3) During his youth he was not exposed to the visual arts. He attended Yale University for two years, but left because he had no interest in academic training.

(New York, Museum of Modern Art, Mark Rothko Exhibit, 18 January 1961)

(4) The connection of the Victoria and Albert Museum with the field of photography, at large as a branch of the visual arts, is not known to a wide public.

(London, Victoria\&Albert Museum, Photographs of Henri Cartier Bresson, 1 January 1969)

The phrase 'visual arts' was introduced during the first half of the $20^{\text {th }}$ century to identify the arts that are primarily visual in nature, such as drawing, painting, sculpting, printmaking, photography, design, crafts, video, filmmaking and architecture, as opposed to music, drama, and literature. Before that, anything that had been created to please the senses - from music to dance, from literature to art as we know it - was commonly gathered under the definition of 'fine arts', which implied an aesthetic judgment and a subtle differentiation between what could be considered fine and what could not.

The phrase 'visual arts' was already in use in art discourse during the time-period covered by the EPA Diacorpus. The Time Magazine Corpus, which can be considered as a valid mirror of the language used by the press from the 1920s onwards, gives evidence of a first occurrence of the phrase in 1932: visual arts are mentioned within an article on the use of art in advertising. Thus, the very origin of the phrase has to be connected with academic environments: the phrase appeared in the title of an influential essay published by the American art historian Bernard Berenson, Aesthetics and History in the Visual Arts (Berenson 1948) and was further consolidated in its use by Erwin Panofsky, who entitled one of his main works Meaning in the visual arts (Panofsky 1955). It is to be noted that the language of EPAs slowly absorbed this new coinage coming from academic settings and has applied it increasingly from the early sixties. Conversely, the phrase fine arts gradually disappeared from the lexis of EPAs, surviving only in crystallized forms, such as the names of museums (for instance, the Boston Museum of Fine Arts), schools, academies, departments, or university courses. There are only 58 occurrences of fine arts within the corpus, most of them (40) dating back to the period 1950-1999.

It might be concluded that EPA writers were responsive to the introduction of new coinages in the field of art discourse, as well as to the decline of other expressions previously in use that may have sounded misleading or out of style. EPA writers were aware of lexical change in their relevant field of action, art discourse, and were also able to apply it to their daily professional language. This result also highlights an urge to discard ambiguous expressions in favor of a more conventional, neutral and specialized language for EPAs, capable of making EPAs more recognizable as a genre within the general domain of art discourse.

\subsection{3. photography}

The increase in the frequency of the noun photography, in parallel to the irregular frequency profile of architecture, can be read as a reflection of the emergence of new visual languages, acknowledged as art forms by museums and audiences, but also as a sign of changing taste of both museum visitors and curatorial staff.

The noun photography ranks $3^{\text {rd }}$ within the top twenty of the most increasing words across decades according to Kendall's tau coefficient and $12^{\text {th }}$ according to Hofland and Johansson. Its pro- 
file is of $4,22,32,59,71,80$ occurrences. Conversely, architecture ranks $20^{\text {th }}$ within the most decreasing words according to Hofland and Johansson, with an irregular profile of 29, 6, 7, 15, 40, 17 occurrences, which also determines an absence of trend according to Kendall's tau coefficient (0.3). architecture is however interesting to examine in this context.

The acknowledgement of photography as an art is traditionally linked to the work of the American photographer Alfred Stieglitz (1864 - 1946), who struggled to establish photography as a valid form of artistic expression (Rosenblum 2007). Thus, it was only in 1937, when the first major exhibition on photography was organized in the United States by Beaumont Newhall at the Museum of Modern Art ('Photography, 1839- 1937', a staple for the history of this medium) that photography gained widespread recognition as an art. A year later, Newhall was appointed first curator of photography and a Department of Photography was founded at the Museum of Modern Art: this was a further tribute and a sign of official recognition with regard to this medium, coming this time from the institutional establishment (Rosenblum 2007). This also explains why photography has been mentioned within American EPAs as a subject for exhibitions since the fifties; conversely, if we look at British EPAs, in the sixties the noun was still used in a completely different sense (5):

(5) Members of the press may preview this Exhibition on Thursday, 29th August from 2.30 to 4.00 p.m. on production of this press notice. Photography is not permitted in the Board Room.

(London, National Gallery, Canaletto Bicentenary Exhibition, 26th August, 1968).

Nonetheless, British museums were becoming aware of the increasing interest in photography, as shown in an EPA issued by the Victoria\&Albert Museum, on the occasion of an exhibition on Henri Cartier-Bresson, which sounds as a sort of justification addressed to the press (6):

(6) The connection of the Victoria and Albert Museum with the field of photography, considered at large as a branch of the visual arts, is not known to a wide public. But its Library houses a notable collection of Victorian photographs; and the Museum mounted the official Centenary exhibition of the Invention of Photography in 1939.

(London, Victoria\&Albert Museum, Henri Cartier-Bresson, 1st January, 1969).

The trend related to the noun architecture plays a more significant role in the American section of the EPA Diacorpus, since 100 occurrences of the word out of 114 belong to that part. Concordances show a major interest in this discipline during the nineties, where most of the occurrences of architecture (40) are concentrated, in correspondence with great exhibitions on masters such as Alvar Aalto and Frank Lloyd Wright. The number of concordances falls drastically in the following decade, where only 3 occurrences of architecture as a field of interest for exhibitions were retrieved, although architecture is not the main topic of the show, but rather a marginal component. The gradual disappearance of the noun architecture from 1990 onwards may suggest at least two conclusions: in the first place, it could reflect the growing difficulties, in technical and economical terms, faced by museums in organizing architectural exhibitions, which is a fact, as well as the reduced opportunities to appoint an architect for renovation projects within the museum due to the high costs of these interventions; on the other hand, this trend could be related to the perception of architecture as a very specialized field of interest and therefore as a more complex and less appealing topic for an exhibition addressed to the general public.

\subsection{Decreasing words: picture, shown, shows}

\begin{tabular}{|r|r|r|r|r|r|r|r|}
\hline Word & \multicolumn{1}{|c|}{1950} & \multicolumn{1}{c|}{1960} & \multicolumn{1}{c|}{1970} & \multicolumn{1}{c|}{1980} & \multicolumn{1}{c|}{1990} & \multicolumn{1}{c|}{$\mathbf{2 0 0 0}$} & \multicolumn{1}{c|}{ Total } \\
\hline picture & 96 & 84 & 16 & 1 & 15 & 12 & 224 \\
\hline shown & 95 & 72 & 32 & 27 & 42 & 36 & 304 \\
\hline shows & 29 & 24 & 15 & 7 & 10 & 15 & 100 \\
\hline
\end{tabular}

Table 5. Profile across decades of decreasing words : picture, shown, shows 


\subsection{1. picture}

Coming to the words showing a decreasing pattern over time, one of the most significant cases is that of picture, ranking third in the top twenty of declining words, with a profile over decades of $96,84,16,1,15,12$ occurrences. Its decrease is not easy to explain. Since the 224 total occurrences of picture are evenly distributed across the British and American sections of the EPA Diacorpus, the trend is relevant for both components. Moreover, the noun decreased only in its singular form and not in its plural, which actually increased $(12,14,15,35,29,69)$. If we take a closer look at the concordances of picture, we can observe that in most cases (158 over 224 total occurrences) the noun is modified by a definite determiner such as the or this, thus EPA writers refer to a specific picture. See for instance the following concordances (7):

(7) was old but not original. The picture will be on public exhibition in the reverie. I am convinced that this picture has a charm all its own in this country. It is the only picture by him which is certainly

The gradual decrease of this typical pattern related to picture could be explained in terms of new design criteria. While in the fifties and the sixties the attention was often placed on a single, specific artwork on display (a picture, namely), nowadays exhibitions are massive events featuring many at once. We cannot expect, therefore, a focus on a single, specific item, but rather a general presentation of the works on display or a list of most prominent.

This trend is confirmed by the growth of other plural forms identifying artworks on display within the EPA Diacorpus, such as images $(3,11,8,21,77,90)$, objects $(5,23,10,14,30,43)$ and works $(67,88,119,140,177,292)$. The increasing frequencies of images, objects and works are not necessarily in contrast with those of the respective singular form, at least not in quantitative terms. In fact, when used in the singular form, these nouns seem to lose their concrete meaning and acquire a different sense. Conversely, the noun picture always keeps its concrete meaning in the EPA Diacorpus, both in its singular and its plural form. If we compare the singular image to its plural images, for instance, we can see that the noun is quantitatively increasing $(0,18,4,9,10$, 39 ), but only in a minor part of the cases the singular noun is used in its concrete sense to refer to a specific work on display. Rather, the image in question refers to a picture formed in the mind of the artist or a general opinion about a person, as in the following concordances (8):

(8) the city is celebrated as an image of sparkling modernity whilst

furniture design and enhance the image of the French furniture industry

an interesting disparity between the image of the ancient world that we derive

Speaking, a rapid-fire combination of image processing and ironic, spoken

Object does not seem to play a relevant role in terms of variation, with 40 occurrences and a profile across decades of 7, 15, 4, 1, 9, 4, while work, which increases over time $(144,122,155,140$, $182,241)$, is much more used in a collective sense, in order to address the whole body of work produced by an artist or to identify a group of objects. See for instance the following concordances (9):

(9) the enormous retrospective of the artist's work at the Foundation in 1968. he boldly returned to figurative work in the late 1960s.

widely varied subjects. His work combines visual elegance with a subtly

He says, "If I look back on my work over a period of years

the context of a consistent body of work that critically investigates

We can argue for the trend of increasing plural nouns identifying objects on display as reflective of the rise of large and multi-item exhibitions, which is a real phenomenon occurred in the last decades and still on-going. This result actually reinforces the appropriateness of the method we adopted so far, since data allow us to make statements on cultural change specific to the environment of museums and exhibitions. 


\subsection{2. shown, shows}

Among the most decreasing words in the EPA Diacorpus are two forms related to the lexeme SHOW: shown and shows. The first is clearly the past participle of the verb show, while shows may be the plural of the noun show or the third person of the present tense of the verb. Since the EPA Diacorpus is not tagged, the distinction was made by hand: in 35 occurrences out of 100 shows is the plural of the noun show. It means that almost 2 out of 3 occurrences are related to the verb, which then plays the most important role. We are therefore authorized to consider both entries, shown and shows, as mainly belonging to the verbal derivation of the lexeme SHOW.

Since the action of putting on display something to be judged by the public - i.e. to show it - is typical of exhibitions, the decrease of these words over time is somehow unexpected in an exhibition press releases corpus and needs to be further explained. The first check was on the frequency and consistency profile of both words.

Shown has 304 total occurrences in the corpus, 144 belonging to the American component and 160 to the British component. Its profile is characterized by a rather irregular decrease over the six decades $(95,72,32,27,42,36)$ and its CV score is 53.10. The most frequent three-word cluster related to shown in the corpus is the future construction will be shown. Similarly, shows, with 100 total occurrences (37 British, 63 American), has an irregular decreasing profile $(29,24,15,7,10$, 15 ) and its CV score is 50.15. Typical patterns in the corpus are the exhibition shows, the painting shows, the section shows, followed by descriptions of art works.

I looked at other forms of the verb show in the corpus, in order to find out if there is a general decrease of its use. The frequency list, alphabetically ordered, provided the following entries: show (291), showcase (3), showcases (3), showcasing (4), showed (10), showing (94).

Show is rather consistent in terms of frequency $(68,50,47,35,37,54)$. It has the same ambiguity as shows, but the manual inspection reveals a reverse tendency: two occurrences out of three refer to the noun, which is a synonym of exhibition. The verbal component is therefore less relevant in this case.

The word-family of showcase has 10 total occurrences in the corpus, all concentrated in the 1990s and in the 2000. It is therefore a more recent lexical input and does not tell us much in terms of variation.

Showed is used very little and is even absent in 2000's EPAs (its profile is 4, 1, 1, 3, 1, 0), while showing is more stable across decades $(17,10,19,18,14,16)$.

So far, we could make only educated guesses as to why the verb show is decreasing over time in the EPA Diacorpus. The Time Magazine Corpus was therefore explored for comparison. The past participle of the verb, shown, significantly decreases across decades even in the Time corpus $(1036,1046,809,586,498,408)$, as does the simple past showed $(2889,2145,1590,1370,1029$, 714). Showing also decreases $(1136,1031,820,676,617,472)$. The same is true for the infinite form $(2447,2244,1875,1708,1708,1320)$ and the present third person of the verb $(1144,1180$, $1034,878,1070,742)$. Therefore, the results of the EPA Diacorpus with regard to the verb show and its decreasing tendency over time mirror - obviously in a smaller dimension - those of a wider, general corpus, specialized in the language of news.

As already pointed out, the verb show has two main meanings in the EPA Diacorpus: the first and more relevant is associated with the action of exhibiting something, while the second is functional to descriptions. In 160 out of a total of 570 occurrences identified within the EPA Diacorpus, show is used to introduce the description of an art work: a picture, a portrait, a painting, etc.

If it is hard to explain why the verb show decreases over time in its first, basic meaning, a guess may be formulated with regard to the second function. When describing art works -a picture, for instance - the use of the verb show implies a critical filter between the writer and the reader: adopting a didactic stance, the writer provides his/her own interpretation of the art work in the form of an authoritative and objective statement, which therefore sounds definitive. 
It can be guessed that filtering verbs such as show have been gradually reduced in the language of EPAs in favor of a more direct way of describing art works and a more nuanced critical stance towards them.

The difference between these two different techniques for describing art works - indirect and direct - can be summarized by the following examples $(10,11)$ taken from the EPA Diacorpus:

(10) This skeletal drawing shows us how the artist went about his task, working out the perspective framework with the ruler and pencil and afterwards filling in the details in ink, underneath which the vanishing point and vanishing lines are still clearly visible.

(London, Victoria\&Albert Museum, Drawings by Italian Artists: 1500-1800, 1 June 1959)

(11) The luminous colour and lively brushwork of the picture evoke the hot, hazy atmosphere of a summer afternoon. This is the world of the Impressionists and the scene is uncompromisingly contemporary. But the isolation and gravity of the figures, the scale of the painting and the classical order of the composition come from a very different world - a tradition stretching back to the Renaissance. The result is at once serene and subversive.

(London, National Gallery, Seurat and the Bathers, 1 March 1997)

In the first extract (10) the writer is present in the text in his authorial stance, identified by the pronoun $u s$, establishing common ground between him and his audience. He directs the reader's gaze, guiding him by pointing to some details of the picture and providing a description of the artist's work; the effect is yet explanatory rather than captivating. In the second extract (11), where the description of the art work is offered without a filtering verb, the reader has the impression of really being there, in front of the picture, in a sort of virtual 'walk' through the exhibition.

\subsection{Stable words: landscape, drawings, paintings}

\begin{tabular}{|r|r|r|r|r|r|r|r|}
\hline Word & \multicolumn{1}{|c|}{1950} & \multicolumn{1}{c|}{1960} & \multicolumn{1}{c|}{1970} & \multicolumn{1}{|c|}{1980} & \multicolumn{1}{c|}{1990} & \multicolumn{1}{c|}{$\mathbf{2 0 0 0}$} & \multicolumn{1}{c|}{ Total } \\
\hline landscape & 25 & 27 & 21 & 23 & 28 & 29 & 153 \\
\hline drawings & 86 & 85 & 59 & 60 & 87 & 67 & 444 \\
\hline paintings & 87 & 86 & 105 & 102 & 99 & 94 & 573 \\
\hline
\end{tabular}

Table 6. Profile across decades of stable words : landscape, drawings, paintings

\subsection{1. landscape}

The noun landscape is the second most frequent lockword of the EPA Diacorpus. It has 153 total occurrences and is characterized by a stable profile across decades $(25,27,21,23,28,29)$, which also determines a low variance score (12.09). Two occurrences of the noun out of three belong to the British section of the corpus, so the trend related to this word has to be regarded as more significant for that part. Other occurrences within the same lexeme are landscapes, landscaping, landscapist and landscapists. The plural form of the noun, landscapes, totaling 76 instances, shows an irregular path over decades $(7,15,7,16,10,21)$, while landscaping, landscapist, both appearing in the fifties, and landscapists, appearing in the nineties, are hapax legomena.

Typically, the noun landscape is introduced by an indefinite article and premodified by an attributive adjective carrying evaluative meaning and functional to the description of the picture: it may be, for instance, quoting examples from the corpus, a romantic, a realistic, a fantastic, a luminous or a pure landscape. At least half of all the occurrences can be ascribed to this recurrent construction. If we look at the co-text, it can also be noticed that the word is especially related to the technique of painting: in about 40 occurrences, the noun appears in the immediate surroundings of words like painting/s, painter/s, picture, depicting, and, in fewer cases, drawing/s and photography. Moreover, in 31 occurrences, landscape is part of the title of a picture or of an exhibition, as shown in the following concordances (12): 
(1) of Esaias van de Velde's little "Winter Landscape", which may well have influenced

The Poetic Landscape takes a new look at Claude as a painter

Caspar David Friedrich's Moonlit Landscape, or Turner's The Pass at

It turns out that the word landscape is used in consideration of its descriptive force, which can be enhanced through evaluative adjectives. Moreover, the noun evokes a well-known genre in the field of visual arts, which everyone is familiar with. However, these elements alone do not explain the persistence and the stability of the noun landscape in the language of EPAs from the fifties onwards. An intriguing suggestion may be that, although new art techniques were introduced and new media emerged in the last decades, such as photography, film and video, a landscape remained a consistent object in art displays, a sort of classic, an evergreen that never grows old. At this point, it could be objected that other subjects can be regarded as fashionless and out of time in art as well: portraiture, in particular, has always been the great alternative to landscape painting.

The portrait or landscape question is important because it defines the place and role of people in a picture. In a portrait, one encounters a person and is introduced to a sort of conversation between the painter and the model. A landscape offers a different experience: figures may be included, but at a greater distance from the artist and viewer. This offers a more circumspect view of people.

Consequently, the EPA Diacorpus was explored in search of related to the lexical field of portraiture. While the use of the verb portray is rather irrelevant - less than 10 overall occurrences - the word portrait (211 total occurrences) shows an irregular path over decades $(28,56,18,45$, $22,42)$, while its plural form, portraits (174 total occurrences), gradually increases $(12,14,15$, $35,29,69)$. The noun portraiture identifying the practice of making portraits is increasing as well $(5,0,1,5,9,12)$. This may reflect a fluctuation in the preferences of exhibitions' visitors and curators, gradually shifting their attention towards individuals, to be approached from a closer point of view, in a sort of voyeuristic curiosity that is typical of our times. The clear growth of the plural form, portraits, opposed to the irregular one shown by the singular, portrait, can also be related to the proliferation of multi-item exhibition, another phenomenon which has emerged in contemporary times (see also the case of picture vs. pictures in section 5.2).

\subsection{2. drawings, paintings}

Cultural and educational reasons may also be invoked in order to explain the consistency over time of the plural nouns drawings and paintings, which rank respectively $10^{\text {th }}$ and $14^{\text {th }}$ among the lockwords of the EPA Diacorpus. The profiles of these words across decades are respectively 86, $85,59,60,87,67$ and 87, 86, 106, 102, 99, 94.

A peculiarity of these words is that they often appear together as a binomial - paintings and drawings (18 occurrences) - or in combination with other nouns: drawings and prints (24), paintings and sculptures (13), prints and drawings (11), paintings, drawings and sculpture (10).

Corpus evidence in this case reflects how art works were and still are combined within the exhibition design in order to provide a comprehensive overview of artists or art movements. Sketches and drawings, for instance, can be used to explain the preliminary work behind a painting and to show how the artist developed his original idea through a series of gradual steps. Such an approach adds value to the exhibition's experience and allows curators, at the same time, to enrich the exhibit itself with more items on display. The following extract (13) provides a relevant example of this design strategy:

(2) Andrea Mantegna (c. 1431 - 1506) was one of the greatest artists of the early Italian Renaissance. This exhibition, in which paintings, drawings and engravings have been assembled from throughout the artist's career, traces the development of Mantegna's innovative genius.

(London, Royal Academy of Arts, Andrea Mantegna, 1 January 1992)

The educational purpose associated in showing paintings and drawings together goes hand in hand with the urge to provide a significant volume of pieces on display. Drawings, prints and lith- 
ographs are generally easier and less expensive to transport and therefore to exhibit. In times of financial difficulties, this may also sound as a valid argument.

Moreover, the stability of the nouns paintings and drawings over time can be interpreted as evidence for the stability of these techniques: despite the rise of new media such as photography and video in the second half of the $20^{\text {th }}$ century, painting and drawing still remain a relevant feature. Other words related to the lexeme PAINT show a stable or even an increasing profile across decades, as in the case of painted $(23,46,7,22,20,47)$, painting $(97,89,78,75,77,145)$, painter $(21,30,12,26,27,26)$. Similarly, words belonging to the lexeme DRAW have a consistent profile, such as drawing $(16,19,12,11,25,28)$ and $\operatorname{draw}(2,2,3,2,2,8)$.

A special case is that of painters, which, on the other hand, ranks $13^{\text {th }}$ within the top list of decreasing words of the corpus $(38,15,17,26,19,24)$. The reason for this decrease could be related to the system of values shared by art professionals, which shapes EPAs and substantiates many lexical choices made by writers. In our times, to define artists merely as painters could sound limiting or, at least, too technical, as contemporary artists can often manage different media and several techniques at the same time. This is a consequence of contemporary art, where the boundaries between the traditional media categories - painting, sculpture, photography etc. - have become blurred. When talking about their roles, artists themselves avoid strict definitions. It is to be noted that the noun sculptors also decreases over time within the EPA Diacorpus, falling from 17 to 8 occurrences, while photographers increases (from 6 to 30 occurrences), maybe as a consequence of the emergence of photography as a new medium. The singular forms painter, photographer and sculptor show different profiles across decades: painter is stable, photographer increases, sculptor decreases. Conversely, the occurrences of artist and artists increase over decades. Such contrasting data do not allow us to make general and definite claims in this direction. Nonetheless, three out of six nouns identifying artists on the basis of a specific skill show a clear pattern of decrease within the EPA Diacorpus. This leads us to suggest an on-going lexical change, maybe not completed yet: a trend reflective of how artists see themselves and want to be seen and presented to the press, not merely as masters of a technique, but rather as creative and multifaceted talents.

\section{Conclusion}

One of the aims of this article was to apply Baker's (2011) method to identify variation over time on a multiple corpus of the specialized language of art discourse, in order to find out if it could bring about interesting results not only in terms of language variation, but also in terms of cultural change related to the field of exhibitions, museums and art in general from 1950 onwards. We can conclude that the method has proved valuable, as it has allowed us to report a number of lexical trends reflective of changes in the way exhibitions are organized and communicated to the public.

In particular, lexical change has revealed some innovations in the way exhibitions are set up and artists are selected by museum professionals. With regard to exhibition design, lexical variation suggests a trend towards comprehensiveness rather than selectiveness. Large exhibits are preferred to narrowly focused events, while increasing care is taken in evaluating and choosing artists, preferably avoiding the risk to present new and emerging names.

In addition, the taste of visitors changes fast and museums have to deal with these fluctuations. Our analysis revealed that from 1950 onwards new media have emerged in the visual arts, but they did not seriously challenge classic subjects and topics of exhibitions.

A significant phenomenon reflected by lexical variation of EPAs is the shift from one-item to multi-item exhibitions: the decline of the word picture in its singular form rather than its plural, typically pre-modified by a determiner (the, this), in parallel to the increase of many other plural nouns identifying art works to put on display (images, works, objects) leads us to that conclusion. Moreover, something has changed in the way artists are selected for exhibiting: they do not have to be famous - another declining word -, nor particularly great or known, since these adjectives are preferably used to refer to their work, but they definitely must have an acknowledged career. 
Indeed, young artists have fewer advantages in the art scene, as reflected by the lexical choice of EPAs, where less than 30 occurrences can be ascribed to the semantic field of youth.

Art subjects may change, and portraits - an increasing item within the EPA Diacorpus - may be more intriguing for our times, but a landscape is still a must for any exhibition, as well as drawings and paintings - to mention some of the lockwords of the corpus - especially if the latter are combined in order to enrich the educational experience of visitors, as well as increase the number of art works on display with a close eye on costs. New media have emerged over time: corpus evidence shows the rise of photography and the decline of architecture. However, the art of painting and drawing has not gone out of style, as words belonging to both lexemes, PAINT and DRAW, show a stable pattern across decades.

As regards the register of EPAs, the analysis of variation has highlighted a preference for more vivid and straightforward descriptions: the decline of the verb shown and shows, to be seen as a general trend of language when compared with the Time Magazine corpus, can be interpreted in these terms. Moreover, the language of EPAs has become more accurate over time, showing an increasing awareness of new coinages in the field of art discourse, such as the phrase visual arts, gradually borrowed from academic settings, in parallel to the dismissal of fine arts. EPA writers also seem responsive to the way artists prefer to be presented to the public, not merely as painters, for instance, to mention another decreasing item, but rather in a more complex light, as implied by the definition of artist and artists, which are both increasing words.

Let us go back to Irvine's (2004-2009) definition of art discourse as something "defining the cultural category of art and maintaining the art/non-art binary": the diachronic analysis of EPAs has confirmed that statement, showing how EPA writers are involved in the process of shaping the concept of art and the ever-changing values related to exhibitions through their lexical selection. The results of the analysis, therefore, highlight the significant role EPAs play within art discourse.

\section{References}

Atkins, Robert 1990: Artspeak. New York: Abbeville Press Publisher.

Baker, Paul 2010: Will $M s$ ever be as frequent as $M r$ ? A corpus-based examination of gendered terms across four diachronic corpora. In Language and Gender 4 (1), 125-149.

Baker, Paul 2011: Times May Change, But We Will Always Have Money: Diachronic Variation in Recent British English. In Journal of English Linguistics 39 (1), 65-88.

Berenson, Bernard 1948: Aesthetics and history in the visual arts. New York: Pantheon.

Bondi, Marina 2013: Historians as Recounters: Description across Genres. In Gotti, Maurizio/Guinda, Carmen Sancho (eds.), Narratives in Academic and Professional Genres. Bern: Peter Lang, 1-25.

Buchloh, Benjamin H.D. 2001: Andy Warhol's One-Dimensional Art: 1956-1966, In Michelson, Annette (ed.), Andy Warhol. Cambridge, MA: MIT Press.

Carrier, David 1987: Artwriting. Amherst: University of Massachusetts Press.

Conboy, Martin 2011: The Language of Newspapers. Socio-historical perspectives. London: Continuum.

Davies, Mark 2007: TIME Magazine Corpus [online]. http://corpus.byu.edu/time (accessed 1 August 2013).

Duguid, Alison 2010: Newspaper discourse informalisation: a diachronic comparison from keywords. In Corpora 5 (2), 109-138.

Harris, Roy 2003: The Necessity of Artspeak: the Language of the Arts in the Western Tradition. London: Continuum.

Hundt, Marianne/Mair, Christian 1999: “Agile” and "upright” genres: the corpus-based approach to language change in progress'. In International Journal of Corpus Linguistics 4 (2), 221-42.

Jacobi, Daniel/Poli, Marie Sylvie 1995: Scriptovisual documents in exhibitions: some theoretical guidelines In Blais, Andrée (ed.), Text in the Exhibition Medium, Montreal, Société des Musées Quebecois and Musée de la Civilisation, 49-73.

Jacobs, Geert 1999a: Preformulating the News. Amsterdam: Benjamins.

Jacobs, Geert 1999b: Self-reference in press releases. In Journal of Pragmatics 31, 219-242. 
Hilpert, Martin/Stefan Th. Gries 2009: Assessing frequency changes in multi-stage diachronic corpora: applications for historical corpus linguistics and the study of language acquisition. In Literary and Linguistic Computing 34(4). 385-401.

Hilpert, Martin/Stefan Th. Gries: Quantitative approaches to diachronic corpus linguistics. In Kytö, Merja/Pahta, Päivi (eds.), The Cambridge Handbook of English Historical Linguistics. Cambridge: Cambridge University Press (to appear) [online]. http://www.linguistics.ucsb.edu/faculty/stgries/research/overview-research.html (accessed 11 December 2013)

Hofland, Knut/Johansson, Stig 1982: Word frequencies in British and American English. Bergen: Norwegian Computing Centre for the Humanities.

Irvine, Martin 2004-2009: Approaches to the Art Media: Modes of Art Talk, Discourses, and the Construction of Art as an Object. http://www9.georgetown.edu/faculty/irvinem/CCTP738/ArtMediaTheory.html (accessed 22 September 2012).

Lazzeretti, Cecilia/Marina Bondi 2012: 'A hypnotic viewing experience'. Promotional features in the language of exhibition press announcements. In Pragmatics 22:3, 567-589.

MacLean, Fiona 1997: Marketing the museum. New York: Routledge.

McCarthy, Kevin F. / Brooks, Arthur / Lowell, Julia F. / Zakaras, Laura 2001: The Performing Arts in a New Era. Santa Monica, CA: RAND Corporation. http://www.rand.org/pubs/monograph_reports/MR1367 (accessed 10 December 2013).

McEnery, Tony/Xiao, Richard/Tono, Yukio 2006: Corpus-based Language Studies: An Advanced Resource Book. London/New York: Routledge.

McManus, Paulette 2000: Archaeological displays and the public: museology and interpretation. London: Archetype Publications.

Panofsky, Erwin 1955: Meaning in the VisualArts. Chicago: The University of Chicago Press.

Partington, Alan (ed) 2010: Modern Diachronic Corpus-Assisted Discourse Studies. Edinburgh: Edinburgh University Press.

Partington, Alan 2012: The changing discourses on antisemitism in the UK press from 1993 to 2009: A modern-diachronic corpus-assisted discourse study. In Journal of Language and Politics 11:1, 51-76.

Radighieri, Sara 2009: Spatial description in the art exhibition review. In Radighieri, Sara/Tucker, Paul (eds.), Point of View: Description and Evaluation across Discourses. Roma: Officina Edizioni, 97-112.

Ravelli, Louise J. 2006: Museum Texts: Communication Frameworks. London: Routledge.

Rosenblum, Naomi 2007: A World History of Photography. New York: Abbeville Press Publishers.

Schiele, Bernard 1995: Text in the exhibition medium.. In Blais, Andrée (ed.), Text in the Exhibition Medium, Montreal, Société des Musées Quebecois and Musée de la Civilisation, 33-47.

Scott, Mike 2007: WordSmith tools 5.0 [online]. http://www.lexically.net/wordsmith/version5/index.html (accessed 1 August 2013).

Tognini-Bonelli, E.: 2001. Corpus Linguistics at Work. Amsterdam/Philadelphia: John Benjamins. 